\title{
An Output Force Control for Robotic Manipulator by Changing the Spring Stiffness
}

\author{
Jirong Wang, ${ }^{1}$ Yuhang Zheng $\mathbb{D}^{1},{ }^{1}$ Jun Li, ${ }^{2}$ Cheng Liu, ${ }^{1}$ Youliang Huang, ${ }^{1}$ and Yu Liu ${ }^{1}$ \\ ${ }^{1}$ College of Mechanical and Electronic Engineering, Qingdao University, Qingdao 266300, China \\ ${ }^{2}$ College of Computer Science and Technology, Qingdao University, Qingdao 266300, China \\ Correspondence should be addressed to Yuhang Zheng; 2498365119@qq.com
}

Received 4 December 2019; Revised 10 January 2020; Accepted 3 February 2020; Published 24 April 2020

Guest Editor: Jing $\mathrm{Na}$

Copyright (c) 2020 Jirong Wang et al. This is an open access article distributed under the Creative Commons Attribution License, which permits unrestricted use, distribution, and reproduction in any medium, provided the original work is properly cited.

This paper presents an output control for a manipulator by changing the spring stiffness. Through the modeling and analysis of the nonlinear stiffness characteristics of the crank-rocker mechanism, and using the zero stiffness domain search method to select the appropriate spring stiffness, using different spring stiffness to establish different mechanism models, the robot can finally control the output of ideal constant force, and at the same time, the analysis results are applied to the improved design of the tire grabbing manipulator. Through this method, the tire grabbing manipulator becomes a constant grabbing force mechanism, and the mechanism is transformed from a rigid-body mechanism to a pseudo-rigid-body mechanism. The accuracy and stability of the whole system are greatly improved. In this study, the method of adding spring to each joint of the linkage mechanism is applied to the improvement design of the linkage mechanism, and the four-bar constant force mechanism is designed for the first time, which expands the application field of the nonlinear stiffness characteristics of the linkage mechanism, and has great application value to the improvement design of the mechanical system with the linkage mechanism and the control of the output force.

\section{Introduction}

Handling robots are widely used in machine tool loading and unloading, stamping machine automatic production line, automatic assembly line, palletizing, and container and other automatic handling. In the tyre industry, the tyre handling robot can not only make full use of the space of the environment but also can improve the material handling capacity, save the working time in the process of loading and unloading, and improve the efficiency of loading and unloading. The weight of all-steel radial tyres is generally heavy. According to the size parameters of the all-steel radial tyre, the overall design of the triple-speed truss manipulator is carried out.

The designed manipulator can realize the technological actions of grabbing tyres for the assembly line and completing tyre vulcanization, loading pot, and storage and stacking. But in the actual operation process, because of the inadequate motion accuracy and the excessive grasping force of the manipulator, it is easy to cause the appearance defects of the tyre bead when the manipulator grasps the tyre, which not only affects the safety of the tyre but also affects the installation of the tyre and other processes. If the gripping force of the manipulator is too large, the deformation of the tyre blank and ellipticity of the tyre will increase during storage and stacking, which will cause the chuck to gnaw the steel ring in the vulcanization process and lead to the extrusion of the rubber and the outcrop of the tyre rim. The vibration of the manipulator in the process of operation can easily lead to the accumulation of errors, which will eventually lead to a large deviation of the tyre stacking position. At the same time, because the grasping mechanism of the manipulator is a rigid-body mechanism, there is a large friction and clearance itself, which will also lead to the problem of tyre stacking position. When the tyre is stored in a vertical tyre storage truck, once there is a problem in the storage location, there are also some problems such as the deformation of the blank and the ellipticity of the tyre, which will also lead to extrusion of the compound and the appearance of the bead. The appearance defect of the tyre bead 
will seriously affect the qualified rate of the tyre, reduce production efficiency, and increase production cost.

In view of the problem that the grasping force of the manipulator is too large, the traditional solution is to calculate the theoretical diameter of the tyre bead according to the product design and component size and, at the same time, to measure the actual diameter of the tyre bead after forming. Based on the minimum diameter, determination of the maximum distance between the claws after the manipulator is closed was performed. Then, comparing the distance between the claw pieces after closing of the current manipulator, cutting and grinding the excess parts of the claw pieces, testing the safety of different quality tyres after lifting, ensuring that the tyre will not squeeze the bead when the manipulator grabs the tyre, and that the bead will not fall off after lifting, and solving the problem of the upper bead crack caused by the extrusion of the mechanical claw pieces on the bead were performed. However, this improvement method is too cumbersome and unsuitable for tyre production with different inner diameters. Therefore, in order to solve the problem of excessive grasping force and tyre production with different inner diameters, the constant force grasping mechanism manipulator is used to solve the aforementioned problems.

Using the characteristics of the mechanical structure, the constant force of mechanisms can provide approximately constant force output. Constant force of mechanisms is widely used in overload protection, biomedical applications, and robotic end effectors to provide friendly interaction with the environment, as well as to protect micro-objects from damage to micro-operations [1]. Because of its many advantages, many scholars have carried out a lot of research on it. Guimin Chen optimized the parameters of the constant force mechanism by using the nonlinear stiffness characteristics [2]. Chia-Wenhou proposed a functional joint mechanism with constant torque output, studied the torque speed curves of different materials, and compared their resistance to hysteresis and stress relaxation [3]. Patrice Lambert proposed a new type of constant force mechanism of pin shaft connection and analyzed the influence of friction produced by pin shaft connection on output force by using the establishment of nonlinear stiffness [4]. Piyu Wang designed a XY precision positioning platform with constant force output parallel kinematics based on a compact flexible mechanism, which can generate constant output force without using a force controller [5]. Tolman proposed a fully flexible constant force mechanism with the initial angle parallel guiding mechanism. Using the finite element model and the experimental prototype test, the pseudo-rigid-body model of the mechanism was established and verified [6]. Liu introduces the design and control of a new passive flexible constant force fixture. The positive stiffness mechanism and negative stiffness mechanism are combined to realize the constant force output function, and the validity of the fixture system is verified through the experimental study of the microcopper wire grip release operation [7]. For the application of the constant force mechanism in industry, the most representative is the constant force compression mechanism. Constant force compression mechanism
(CFCM) is a kind of slider mechanism with large deflection beam. Midha et al. first introduced this mechanism and weight and proposed the selection method of this mechanism [8]. Later, Boyle [9] developed a dynamic model of the flexible constant force compression mechanism. It is necessary to establish the nonlinear stiffness characteristics when the constant force of mechanism is designed. The research and application of the nonlinear stiffness characteristics of compliant mechanisms are also very extensive. Zheng constructed high-static and low-dynamic-stiffness (HSLD) pillars with negative stiffness magnetic spring (NSMS), established a Stewart isolation platform model with high strength steel braces, and studied the stiffness characteristics of the isolation platform [10]. Zongwei Yang improved the weight balance mechanism by synthesizing the required nonlinear torque curve with two linear springs (one tension spring and one compression spring) [11]. Andò presented a low-cost vibration energy acquisition method based on the nonlinear stiffness design of springs [12]. Lucas studied the effect of replacing the main spring with a nonlinear cubic spring. It is proved that if the stiffness is softened, the use of cubic stiffness can improve the frequency transfer rate around and above the resonance [13]. Through static analysis of the nonlinear stiffness system, Wu proved that the nonlinear stiffness system can improve the efficiency of wave energy collection and increase the average power by improving the inherent period and expanding resonance [14]. Ivana Koavcic studied a vibration isolator consisting of a vertical linear spring and two nonlinear prestressing oblique springs [15]. Based on a free vibration test, Bin Tang simply estimated the performance of the absorber by connecting the nonlinear absorber with the vibrating screen [16]. Pellegrini studied an alternative method of obtaining mechanical vibration by using two kinds of energy collectors with stable structures. According to the feasibility of miniaturization, different designs and classifications of existing bistable energy collectors were made [17]. Xiuting Sun studied an N-story shear structure (SLS) vibration isolation platform, focusing on the analysis and design of its nonlinear stiffness, friction, and damping characteristics, and obtained good vibration isolation performance [18]. The constant force grasping mechanism manipulator can achieve the function of grasping tyre well, and because the grasping force is constant, there will be no case of extrusion of the bead or tyre deformation.

\section{Grasping Mechanism of Tyre Grabbing Manipulator}

The grabbing mechanism of the triple-speed truss manipulator adopts crank-rocker mechanism (as shown in Figure 1). The lengths of the four connecting rods are $\mathrm{AB}=100 \mathrm{~mm}, \quad \mathrm{BC}=110 \mathrm{~mm}, \quad \mathrm{CD}=130 \mathrm{~mm}, \quad$ and $\mathrm{AD}=180 \mathrm{~mm}$. The angle between $\mathrm{CD}$ and $\mathrm{DE}$ is $50^{\circ}$. The whole manipulator is composed of three identical crankrocker mechanisms (as shown in Figure 2). The power source is the motor. In practical use, the connecting rod $\mathrm{AB}$ of the three linkage mechanisms is simplified as a whole 


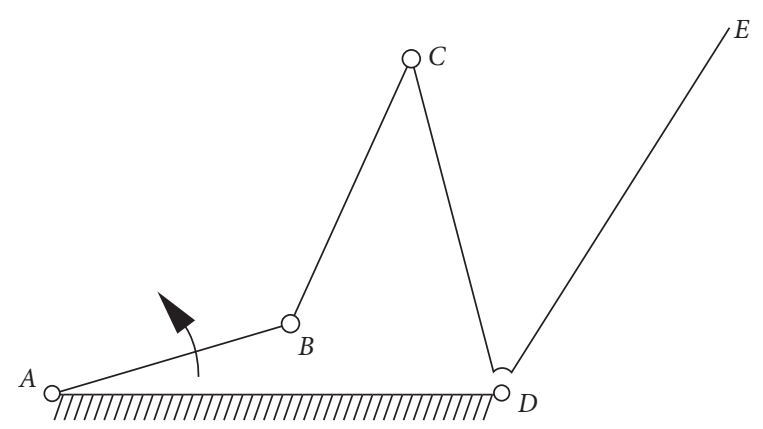

Figure 1: Motion sketch of the mechanism.

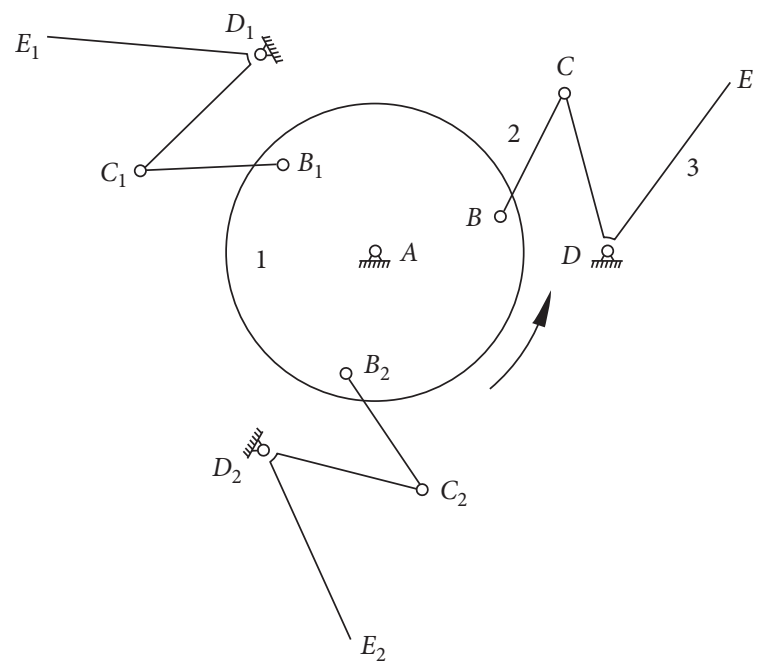

Figure 2: Motion and structure sketch of the manipulator.

into a disc. After the motor passes through the reducer, it is connected with the disc through the coupling and then drives the connecting rod 2 and 3 to rotate. By adjusting the rotation angle of the motor, the crank rotates at the corresponding angle and finally drives the rocker to rotate. The end of point E contacts the tire, and the tire is grasped by three-point positioning.

The three-dimensional structure of the manipulator is shown in Figure 3. Structures 1, 2, and 3 are exactly the same three crank-rocker mechanisms. After calculation, the maximum inner diameter of the tyre grasped by the manipulator is as follows: $\varphi_{d}=609.6 \mathrm{~mm}$. At this time, the maximum output torque of the motor after passing through the reducer is $T_{1}=55.3779 \mathrm{~N} \cdot \mathrm{m}$. The minimum inner diameter of the tyre grasped by the manipulator is $\varphi_{d}=406.4 \mathrm{~mm}$, and the maximum output torque of the motor after passing through the reducer is $T_{2}=104.4972 \mathrm{~N} \cdot \mathrm{m}$.Because the crank-rocker mechanism is a rigid-body mechanism, there are still large clearances and friction, which are prone to errors in the operation of the mechanism. When the equipment decelerates to the left or accelerates to the right, mechanism 3 will be impacted by inertia. When the equipment decelerates to the right or accelerates to the left, mechanism 1 and mechanism 2 will be impacted by inertia, which will also affect the accuracy of the equipment.

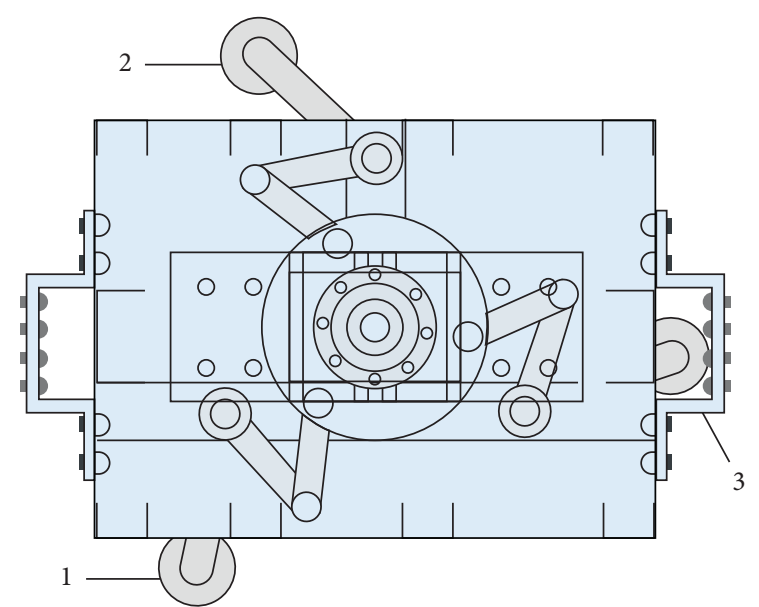

FIgURE 3: Three-dimensional structure of the manipulator.

\section{Tyre Constant Force Grabbing Manipulator}

To solve the problem of tyre deformation, the tyre grasping mechanism can be improved into a constant force mechanism. There are two ways to improve the mechanism into a constant force mechanism, namely, adding force sensors and controllers to the force control technology and transforming the mechanism into a constant force clamping mechanism. At present, many experts are also actively studying new controllers. Shubo Wang proposed an adaptive funnel control (FC) scheme for the unknown dead zone servo mechanism [19] and proposed an unknown input observer for the servo mechanism with unknown dynamics (such as nonlinear friction, parameter uncertainty, and external interference) [20] and designed a new adaptive controller to compensate nonlinear friction and bounded interference [21]. Although the force control technology of adding force sensors and controllers is stable and reliable, the algorithm design is complex and the cost is high. The mechanism improvement is used to transform the mechanism into a constant force mechanism with low cost and simpler improvement. Therefore, the tyre grasping mechanism is improved to a constant force mechanism with the improvement of the mechanical structure. Through consulting the data, it is found that, in the books of Alabuzhev et al. [22], the constant force mechanism (or quasizero stiffness mechanism) can be divided into four categories according to its stiffness characteristics. Through comparison, it is found that the curve beam constant force mechanism is the closest to the crankrocker mechanism. The pseudo-rigid-body model method is adopted in the establishment of the kinematics model. The research method provides a way to design the constant force mechanism with crank-rocker mechanism as the model. The pseudo-rigid-body model method is to transform the constant force mechanism of curved beam into a pseudo-rigid-body mechanism for analysis, as shown in Figure 4. It can be seen from the figure that the transformed mechanism is a pseudo-rigid-body mechanism with spring installed at the node of the mechanism. 


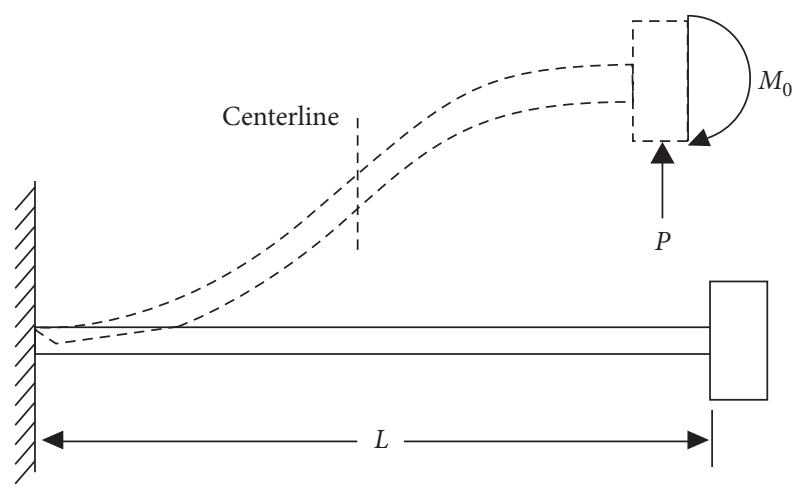

(a)

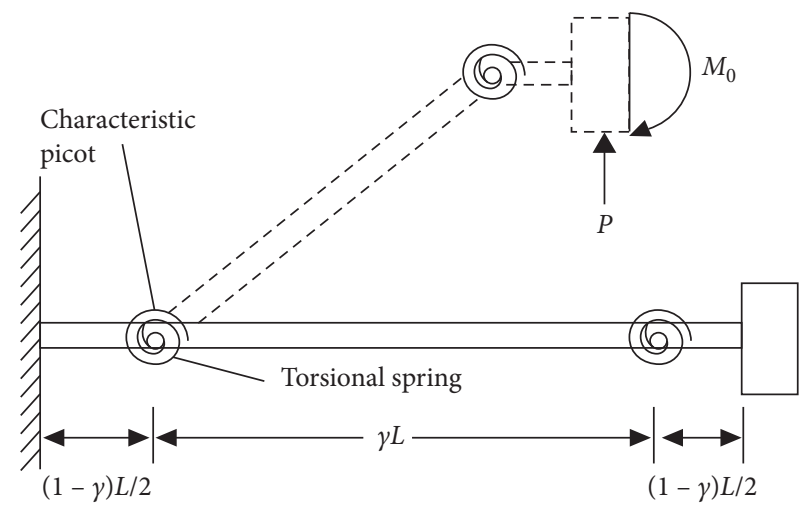

(b)

FIGURE 4: Schematic diagram of (a) fixed-guided beam and (b) the corresponding pseudo-rigid-body method [23].

Through the aforementioned ideas, the joints of crankrocker mechanism can also be installed with springs. Through the stiffness design of the springs, a new constant force mechanism can be designed. A new type of doubleslider mechanism proposed by Baokun Li [24] was very similar to the crank-rocker mechanism, but if the doubleslider mechanism was applied to the improvement of the tyre grasping manipulator, it is necessary to change the length of the connecting rod and to increase the slider. Not only the calculation is difficult but also the improved structure is too large, and the three mechanical structures will interfere. Therefore, the torsion spring can be installed on the node of the crank-rocker mechanism to design the local constant force mechanism. The constant force mechanism designed by this method can not only keep constant grasping force to avoid tyre deformation but also can greatly reduce friction and clearance and extend the life of the machine as the grasping mechanism transforms from a rigid-body mechanism to a pseudorigid-body mechanism after the node is installed with spring.

\section{Dynamic and Static Models of Mechanisms}

Figure 5 shows the mechanism motion sketch of the manipulator. The crank $\mathrm{AB}$ rotates counterclockwise around point $A$ and drives the rocker $\mathrm{CDE}$ to rotate. Crank $\mathrm{AB}$ and rocker $\mathrm{CDE}$ are connected by coupler $\mathrm{BC}$. The torsional stiffness of four joints are $K_{\mathrm{RA}}, K_{\mathrm{RB}}, K_{\mathrm{RC}}$, and $K_{\mathrm{RD}}$, respectively. The stiffness of E-point compression spring is set to $K_{\mathrm{PE}}$.

The Cartesian coordinate system $O-X Y Z$ is fixed to point $A$. The origin $O$ coincides with point $A$. The positive direction of the $x$-axis points to the right side of the horizontal plane. The positive direction of the $y$-axis is vertical. The $z$ axis is determined by the right manipulation.

Vectors $\mathrm{AB}, \mathrm{BC}, \mathrm{CD}$, and $\mathrm{DE}$ are defined by $r_{1}, r_{2}, r_{3}$, and $r_{5}$, respectively. The position $D$ on the $x$-axis is represented by $r_{4}: r_{1}=100 \mathrm{~mm}, \quad r_{2}=110 \mathrm{~mm}, \quad r_{3}=130 \mathrm{~mm}$,

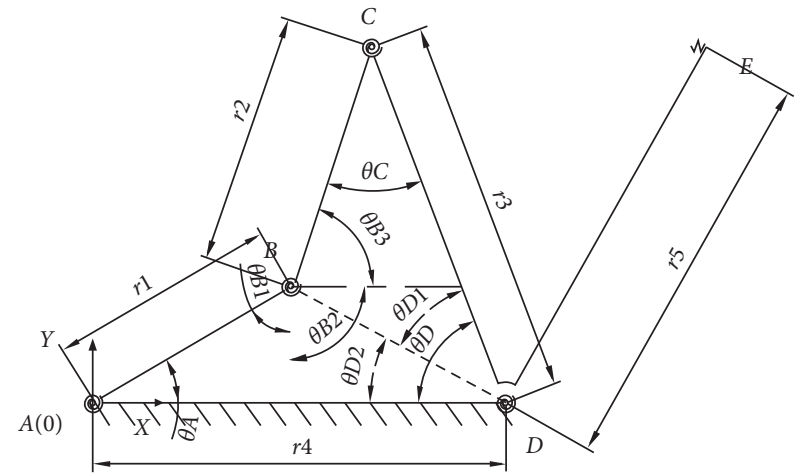

Figure 5: Motion sketch of the manipulator mechanism.

$r_{5}=165 \mathrm{~mm}$, and $r_{4}=180 \mathrm{~mm}$. The angle between CD and $\mathrm{DE}$ is $50^{\circ}$.

In order to show the correct limit position of the mechanism, we assume that there is no friction and clearance between two connecting rods connected by the kinematic pair. In addition, we only discuss the static model of the mechanism in motion, without considering any inertial force (torque) and gravity.

In order to compute the static relationship among the components of the mechanism, it is necessary to first calculate the relationship among the angles. Figure 5 shows that

$$
\begin{aligned}
\theta_{A}+\theta_{B}+\theta_{C}+\theta_{D} & =2 \pi, \\
\theta_{D} & =\theta_{D 1}+\theta_{D 2} .
\end{aligned}
$$

Connect $\mathrm{BD}$ and suppose the length is $a$. According to the cosine theorem,

$$
\begin{aligned}
a^{2} & =r_{1}^{2}+r_{4}^{2}-2 r_{1} r_{4} \cos \theta_{A}, \\
r_{2}^{2} & =a^{2}+r_{3}^{2}-2 a r_{3} \cos \theta_{D 1}, \\
r_{1} \sin \theta_{A} & =a \cdot \sin \theta_{D} .
\end{aligned}
$$

Therefore, it can be obtained that 


$$
\theta_{D 1}=\arccos \frac{a^{2}+r_{3}^{2}-r_{2}^{2}}{2 a r_{3}}=\arccos \frac{r_{1}^{2}+r_{4}^{2}-2 r_{1} r_{4} \cos \theta_{A}+r_{3}^{2}-r_{2}^{2}}{2 r_{3} \sqrt{r_{1}^{2}+r_{4}^{2}-2 r_{1} r_{4} \cos \theta_{A}}}
$$

$\theta_{D 2}=\arcsin \frac{r_{1} \sin \theta_{A}}{\sqrt{r_{1}^{2}+r_{4}^{2}-2 r_{1} r_{4} \cos \theta_{A}}}$

$$
\begin{aligned}
\theta_{D}= & \theta_{D 1}+\theta_{D 2}=\arccos \frac{r_{1}^{2}+r_{4}^{2}-2 r_{1} r_{4} \cos \theta_{A}+r_{3}^{2}-r_{2}^{2}}{2 r_{3} \sqrt{r_{1}^{2}+r_{4}^{2}-2 r_{1} r_{4} \cos \theta_{A}}} \\
& +\arcsin \frac{r_{1} \sin \theta_{A}}{\sqrt{r_{1}^{2}+r_{4}^{2}-2 r_{1} r_{4} \cos \theta_{A}}}
\end{aligned}
$$$$
\theta_{B}=\pi-\theta_{A}+\arcsin \frac{r_{3} \sin \theta_{D}-r_{1} \sin \theta_{A}}{r_{2}},
$$$$
\theta_{C}=\arccos \frac{r_{1}^{2}+r_{4}^{2}-r_{2}^{2}-r_{3}^{2}-2 r_{1} r_{2} \cos \theta_{A}}{-2 r_{2} r_{3}} \text {. }
$$

\section{Using the Principle of Virtual Work to Calculate the Output Force $F$}

At present, there are two methods to establish the dynamic model of the linkage mechanism, that is, to establish the relationship between generalized force and generalized coordinates by the Lagrange equation and to establish the dynamic and static model of the linkage mechanism by virtual work principle. For the general four-bar mechanism, especially the constant-force compressor mechanism, the Lagrange equation method is generally adopted. But there are two problems for the four-bar mechanism: first, the establishment of the Lagrange equation needs to involve velocity calculation. There are kinematic singularities in the linkage mechanism. When the mechanism reaches the dead point or change point, so-called singular positions, the linear velocity equations of mechanism cannot be derived. The angular velocity of the follower bar other than the original bar can be obtained directly. At this time, the motion of the mechanism is usually considered to be uncertain. The motion analysis of the mechanism in singular position is also rare [25]. Second, in the process of establishing the Lagrange equation, it is necessary to define the mass of the link and other moving parts, while in the kinematic analysis of the linkage mechanism, the mass of the link should be determined. Most of the quality is neglected. Therefore, virtual work principle is used to build the dynamic model.

It can be seen from the four sections that the relationship between the angles of the four-bar mechanism is complex, so the calculation of the joint moment and virtual work of the four-bar mechanism by using the principle of virtual work is seldom used. Although the kinematic equation of the double-slider four-bar mechanism is analyzed by $\mathrm{Li}$ [26], because the angle analysis of the doubleslider four-bar mechanism is different from that of the crank-rocker mechanism, the kinematic equation analysis of the double-slider four-bar mechanism cannot be used. However, the method of nonlinear stiffness analysis can be used for reference, and the moment and virtual work of each joint of the four-bar mechanism can also be calculated.

5.1. Utilizing the Principle of Virtual Work to Calculate the Torque and Virtual Work of Joints. The formula of virtual work principle is

$$
\delta W=\sum_{i} F_{i} \cdot \delta r_{i}+\sum_{i} C_{i} \cdot \delta r_{i}=0,
$$

where $F_{i}$ is the external force and $C_{i}$ is the binding force. In this model, the formula becomes

$$
\delta W=\sum_{i} T_{i} \cdot \delta \theta_{i}+F \cdot \delta r=0 .
$$

Among them, $T_{i}$ is the driving torque applied to the bar $A B$ after the motor is decelerated by the reducer and the torque caused by the springs at each node. $F$ is the clamping force needed to clamp the tyre. The driving torque applied on the $\operatorname{rod} A B$ is set to

$$
T_{d}=T_{d} K
$$

Among them, vector $K$ is the unit vector of $z$-axis (vector $i$ and $j$ are the unit vectors of $x$-axis and $y$-axis, respectively). The orbital vector $T_{d}$ is along the $z$-axis. Scalar $\left\|T_{d}\right\|$ is the magnitude of the driving torque, where $T_{d}>0$ represents the positive direction of $T_{d}$ along the $z$ axis and $T_{d}<0$ corresponds to the negative direction of $T_{d}$ to $z$-axis.

Torque caused by springs on each node can be expressed as

$$
T_{i}=K_{R i} \cdot \psi_{i}
$$

where $K_{R i}$ is the stiffness of the spring added to each node and $\varphi_{i}$ is the angular displacement produced by each node. Since the mechanism has four nodes, formula (17) can be expressed as a fourth-order system:

$$
\left[\begin{array}{cccc}
K_{R A} & 0 & 0 & 0 \\
0 & K_{R B} & 0 & 0 \\
0 & 0 & K_{R C} & 0 \\
0 & 0 & 0 & K_{R D}
\end{array}\right]\left[\begin{array}{c}
\psi_{A} \\
\psi_{B} \\
\psi_{C} \\
\psi_{D}
\end{array}\right]=\left[\begin{array}{c}
T_{A} \\
T_{B} \\
T_{C} \\
T_{D}
\end{array}\right] .
$$

Angular displacement at joint $A$

$$
\psi_{A}=\left(\theta_{A}-\theta_{A 0}\right) K
$$

Among them, $\theta_{A 0}$ is the rotation angle from $x$-axis to connecting rod $A B$, indicating the input position angle of connecting rod $\mathrm{AB}$, and $\theta_{\mathrm{A}}$ corresponds to the initial angle. Here, we consider $\theta_{\mathrm{A}}$ as a general coordinate mechanism. Similarly, the virtual angular displacements relative to joint $B, C$, and $D$ are $\psi_{\mathrm{B}}=\left(\theta_{\mathrm{B}}-\theta_{\mathrm{B} 0}\right) K, \psi_{\mathrm{C}}=\left(\theta_{\mathrm{C}}-\theta_{\mathrm{C} 0}\right) K$, and $\psi_{\mathrm{D}}=\left(\theta_{\mathrm{D}}-\theta_{\mathrm{D} 0}\right) K$.

Therefore, it can be expressed as 


$$
\left[\begin{array}{cccc}
K_{R A} & 0 & 0 & 0 \\
0 & K_{R B} & 0 & 0 \\
0 & 0 & K_{R C} & 0 \\
0 & 0 & 0 & K_{R D}
\end{array}\right]\left[\begin{array}{c}
\theta_{A}-\theta_{A 0} \\
\theta_{B}-\theta_{B 0} \\
\theta_{C}-\theta_{C 0} \\
\theta_{D}-\theta_{D 0}
\end{array}\right] K=\left[\begin{array}{c}
T_{A} \\
T_{B} \\
T_{C} \\
T_{D}
\end{array}\right] .
$$

The virtual angular displacement relative to joint $A$ is

$$
\delta \psi_{A}=\frac{\mathrm{d} \psi_{A}}{\mathrm{~d} \theta_{A}} \delta \theta_{A} .
$$

The virtual angular displacement relative to joint $B$ is

$$
\begin{aligned}
\delta \psi_{B} & =\frac{\mathrm{d} \psi_{B}}{\mathrm{~d} \theta_{A}} \delta \theta_{A}=\frac{\mathrm{d}\left(\theta_{B}-\theta_{B 0}\right)}{\mathrm{d} \theta_{A}} \delta \theta_{A} K=\frac{\mathrm{d} \theta_{B}}{\mathrm{~d} \theta_{A}} \delta \theta_{A} K \\
& =\frac{\mathrm{d}\left(\pi-\theta_{A}+\arcsin \left(r_{3} \sin \theta_{D}-r_{1} \sin \theta_{A} / r_{2}\right)\right)}{\mathrm{d} \theta_{A}} \delta \theta_{A} K .
\end{aligned}
$$

The virtual angular displacement relative to joint $C$ is

$$
\begin{aligned}
\delta \psi_{B} & =\frac{\mathrm{d} \psi_{B}}{\mathrm{~d} \theta_{A}} \delta \theta_{A}=\frac{\mathrm{d}\left(\theta_{B}-\theta_{B 0}\right)}{\mathrm{d} \theta_{A}} \delta \theta_{A} K=\frac{\mathrm{d} \theta_{B}}{\mathrm{~d} \theta_{A}} \delta \theta_{A} K \\
& =\frac{\mathrm{d}\left(\pi-\theta_{A}+\arcsin \left(r_{3} \sin \theta_{D}-r_{1} \sin \theta_{A} / r_{2}\right)\right)}{\mathrm{d} \theta_{A}} \delta \theta_{A} K .
\end{aligned}
$$

The virtual angular displacement relative to joint $D$ is

$$
\delta \psi_{D}=\frac{\mathrm{d} \psi_{D}}{\mathrm{~d} \theta_{A}}=\frac{\mathrm{d}\left(\theta_{D}-\theta_{D 0}\right)}{\mathrm{d} \theta_{A}} \delta \theta_{A}=\frac{\mathrm{d} \theta_{D}}{\mathrm{~d} \theta_{A}} \delta \theta_{A} K .
$$

The virtual displacement at $E$ is

$$
\delta \psi_{E}=r_{5} \sin \left(100^{\circ}-\theta_{D}\right) \frac{\mathrm{d} \theta_{D}}{\mathrm{~d} \theta_{A}} \delta \theta_{A} K .
$$

By combining formulas (11)-(19) and substituting formula (9), we can get

$$
\delta W=\left[\begin{array}{lllll}
\delta \psi_{A} & \delta \psi_{A} & \delta \psi_{B} & \delta \psi_{C} & \delta \psi_{D}
\end{array}\right]\left[\begin{array}{c}
T_{d} \\
-T_{A} \\
-T_{B} \\
-T_{C} \\
-T_{D}
\end{array}\right]+F \cdot \delta \psi_{E}=0 .
$$

After sorting out, we can get

$$
\begin{gathered}
F=\left[\begin{array}{lllll}
\delta \psi_{A} & \delta \psi_{A} & \delta \psi_{B} & \delta \psi_{C} & \delta \psi_{D}
\end{array}\right]\left[\begin{array}{c}
-T_{d} \\
T_{A} \\
T_{B} \\
T_{C} \\
T_{D}
\end{array}\right] \frac{1}{\delta \psi_{E}} \\
=-\frac{T_{d}-K_{R A}\left(\theta_{A}-\theta_{A 0}\right)-K_{R B}\left(\theta_{B}-\theta_{B 0}\right)\left(\mathrm{d} \theta_{B} / \mathrm{d} \theta_{A}\right)-K_{R C}\left(\theta_{C}-\theta_{C 0}\right)\left(\mathrm{d} \theta_{C} / \mathrm{d} \theta_{A}\right)-K_{R D}\left(\theta_{D}-\theta_{D 0}\right)\left(\mathrm{d} \theta_{D} / \mathrm{d} \theta_{A}\right)}{r_{5} \sin \left(100^{\circ}-\theta_{D}\right)\left(\mathrm{d} \theta_{D} / \mathrm{d} \theta_{A}\right)} .
\end{gathered}
$$

It can be seen from formula (21) that the change trend of grasping force $F$ curve with $\theta_{\mathrm{A}}$ is related to the driving moment $T_{d}$ and the torsional stiffness of each torsion spring. In order to set appropriate parameters to obtain constant grasping force, it is necessary to analyze the influence of each parameter on $F$. To analyze the influence of each parameter, one of the torques can be set to nonzero and the other to zero and the grasping force can be used as the output to obtain a fifth-order system: 


$$
F=\left[\begin{array}{l}
F_{d} \\
F_{A} \\
F_{B} \\
F_{C} \\
F_{D}
\end{array}\right]=\left[\begin{array}{l}
\frac{\mathrm{d} \psi_{A}}{\mathrm{~d} \theta_{A}} \\
\frac{\mathrm{d} \psi_{A}}{\mathrm{~d} \theta_{A}} \\
\frac{\mathrm{d} \theta_{B}}{\mathrm{~d} \theta_{A}} \\
\frac{\mathrm{d} \theta_{C}}{\mathrm{~d} \theta_{A}} \\
\frac{\mathrm{d} \theta_{D}}{\mathrm{~d} \theta_{A}}
\end{array}\right]\left[\begin{array}{lllll}
T_{d} & T_{A} & T_{B} & T_{C} & T_{D}
\end{array}\right]
$$

where $F_{d}$ is the grasping force $F$ when the driving moment $T_{d} \neq 0$ and the stiffness of other joint torsion springs is set to $K_{R A}=K_{R B}=K_{R C}=K_{R D}=0$. At this time, the grasping force is

$$
F=F_{d}=-\frac{T_{d}}{r_{5} \sin \left(100^{\circ}-\theta_{D}\right)\left(\mathrm{d} \theta_{D} / \mathrm{d} \theta_{A}\right)} .
$$

Through calculation, it can be concluded that when $\theta_{A}=42.1^{\circ}$, the grasping force $F$ tends to infinity, which is located in the singular position of the linkage. Because it is difficult to analyze the singular position of motion, the position is abandoned. By analyzing other position curves, there is a bistable curve between $-100^{\circ}$ and $-10^{\circ}$, as shown in Figure 6. The analysis shows that the bistable curve is easy to fit other curves. Therefore, we chose this curve to fit.

When the driving torque is $T_{d}=0$, the stiffness of one torsion spring is set as nonzero and that of other joints is set as zero, and

$$
F=F_{i}=\frac{K_{R i}\left(\theta_{i}-\theta_{i 0}\right)}{r_{5} \sin \left(100^{\circ}-\theta_{D}\right)\left(\mathrm{d} \theta_{D} / \mathrm{d} \theta_{A}\right)} .
$$

In the aforementioned formula, $i=A, B, C, D$.

Set $K_{R A}=1 \mathrm{~N} \cdot \mathrm{m}\left({ }^{\circ}\right), K_{R A}=3 \mathrm{~N} \cdot \mathrm{m}\left({ }^{\circ}\right), K_{R A}=5 \mathrm{~N} \cdot \mathrm{m}\left({ }^{\circ}\right)$, and $\theta_{A 0}=30^{\circ}$, and the stiffness of torsion spring of other joints is set to zero. With MATLAB, the image of grasping force $F_{A}$ changing with input position angle $\theta_{\mathrm{A}}$ can be obtained. Similarly, we can get the image of force $F_{B}, F_{C}$, and $F_{D}$, changing with input position angle $\theta_{\mathrm{A}}$ (as shown in Figure 7). Because the fitting range is selected above, $-100^{\circ}$ to $10^{\circ}$, the curve of $-100^{\circ}$ to $10^{\circ}$ is also selected for fitting. Note that when $\theta_{A} \leq 0$, then $\theta_{B}=2 \pi+\theta_{A}-\theta_{C}-\theta_{D}$, which is different from equation (7).

As can be seen from Figure $7(\mathrm{a})$, in the region of $-100^{\circ}$ to $10^{\circ}$, the grabbing force $F$ shows a monotonous decreasing characteristic when only $K_{R A} \neq 0$. As can be seen from Figure 7(b), the grabbing force $F$ is only monotonically decreasing in the $-100^{\circ}$ to $10^{\circ}$ region, but the calculation method of $\theta_{B}$ is different when $\theta_{A} \leq 0$ and $\theta_{A} \geq 0$.

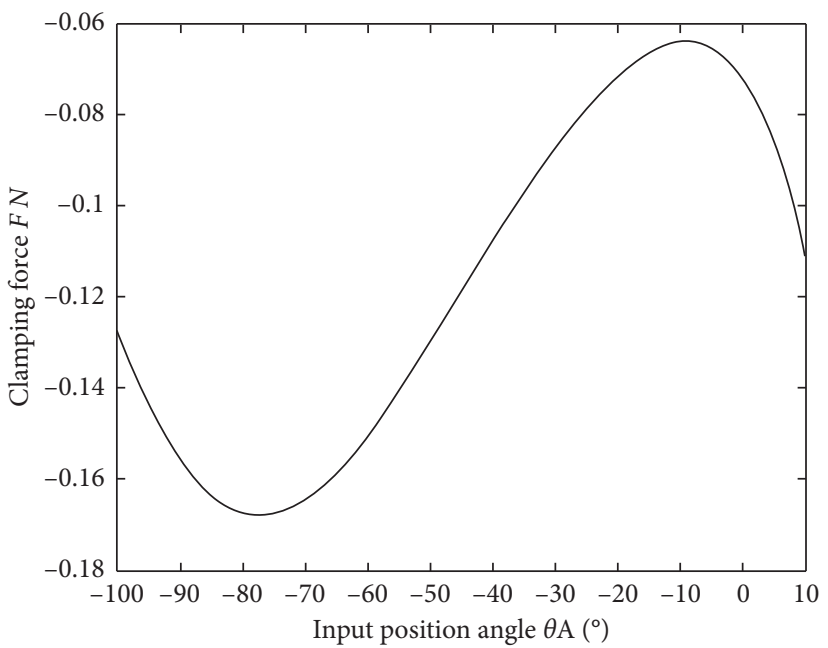

Figure 6: Grasping force $F$ changes with position angle $\theta_{A}$.

Therefore, the torsion spring at joint $B$ is discarded. From Figure 7 (c), it can be seen that, in this region, the grasping force $F$ shows a monotonic decreasing characteristic when only $-100^{\circ}-10^{\circ}$. From Figure $7(\mathrm{~d})$, it can be seen that the grabbing force $F$ increases monotonously when the grabbing force is only $-100^{\circ}$ to $10^{\circ}$ in this region, and it can be found that with the increase of the stiffness of each spring, the image features will be more obvious. Therefore, the desired image features can be obtained by adjusting the spring stiffness properly.

5.2. Determining the Parameters of Each Part and Establishing the Constant Force Interval. According to the curve trend in the previous section, the expected constant force range can be established by designing appropriate spring stiffness. The process of designing the constant force interval is as follows:

(1) Establishing the relationship between spring stiffness: substituting $\theta_{A}=-100^{\circ}$ and other given parameters to solve the differential of equation (22) with respect to $\theta_{\mathrm{A}}$ and then obtaining the following expression:

$$
\left.\frac{\mathrm{d} F}{\mathrm{~d} \theta_{A}}\right|_{\theta_{A}=-100^{\circ}}=f\left(T_{d}, K_{R A}, K_{R B}, K_{R C}, K_{R D}\right)=0 .
$$

(2) Searching constant force interval: shen the grasping force satisfies $|F-F|_{\theta_{A}=-100^{\circ}} /\left.F\right|_{\theta_{A}=-100^{\circ}} \mid \leq 2 \%$, it is considered to be a constant force interval. When the geometric parameters are given as $r_{1}=100 \mathrm{~mm}$, $r_{2}=110 \mathrm{~mm}, r_{3}=130 \mathrm{~mm}, r_{4}=180 \mathrm{~mm}$, and $r_{5}=$ $165 \mathrm{~mm}$ and the initial input position angle is assembled as $\theta_{A 0}=30^{\circ}$, equation (25) is obtained:

$$
0.2347 T_{d}+0.4066 K_{R A}+0.0204 K_{R C}+0.0445 K_{R D}=0 .
$$




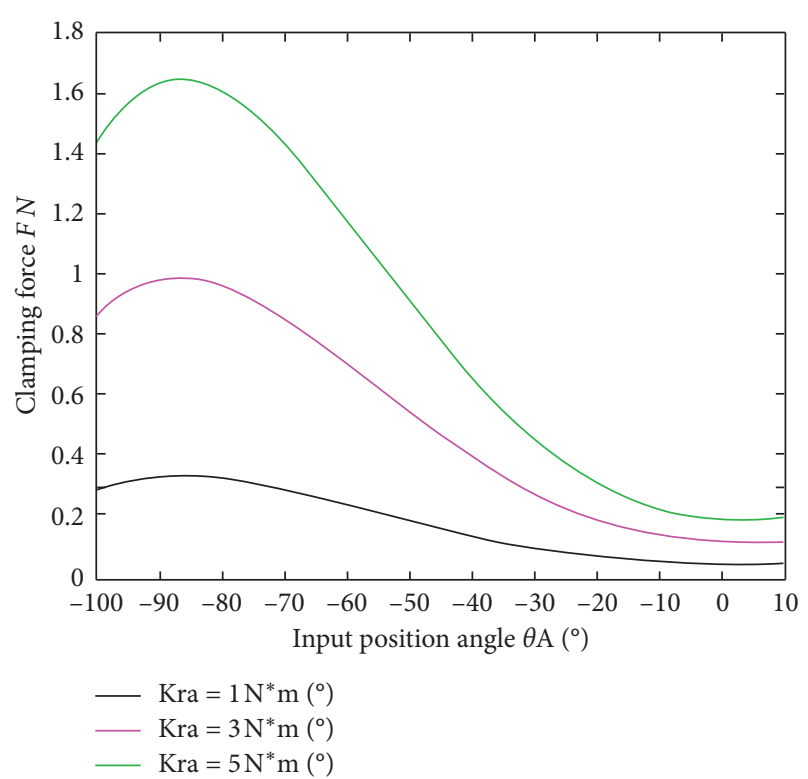

(a)

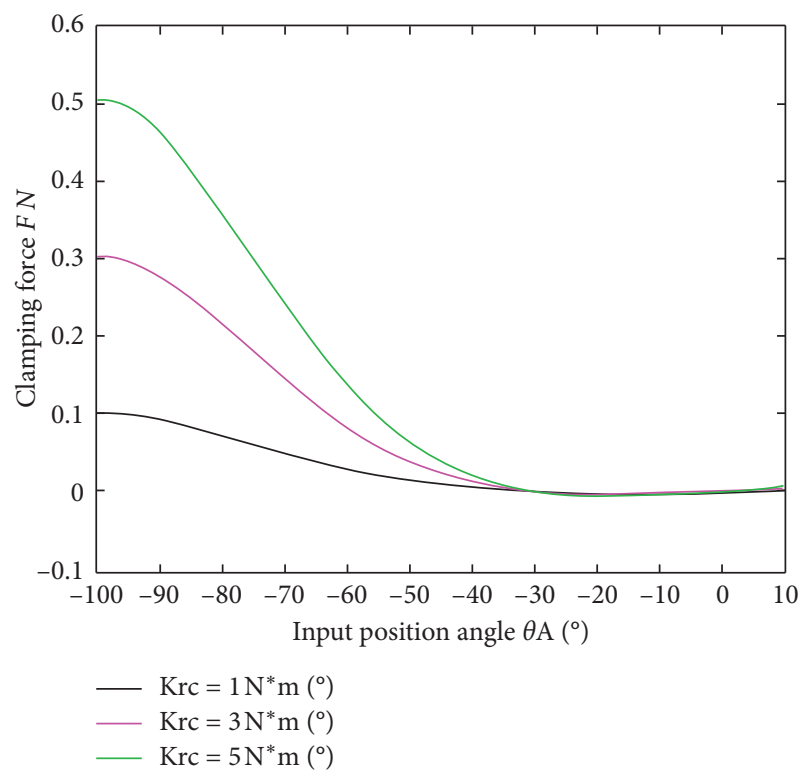

(c)

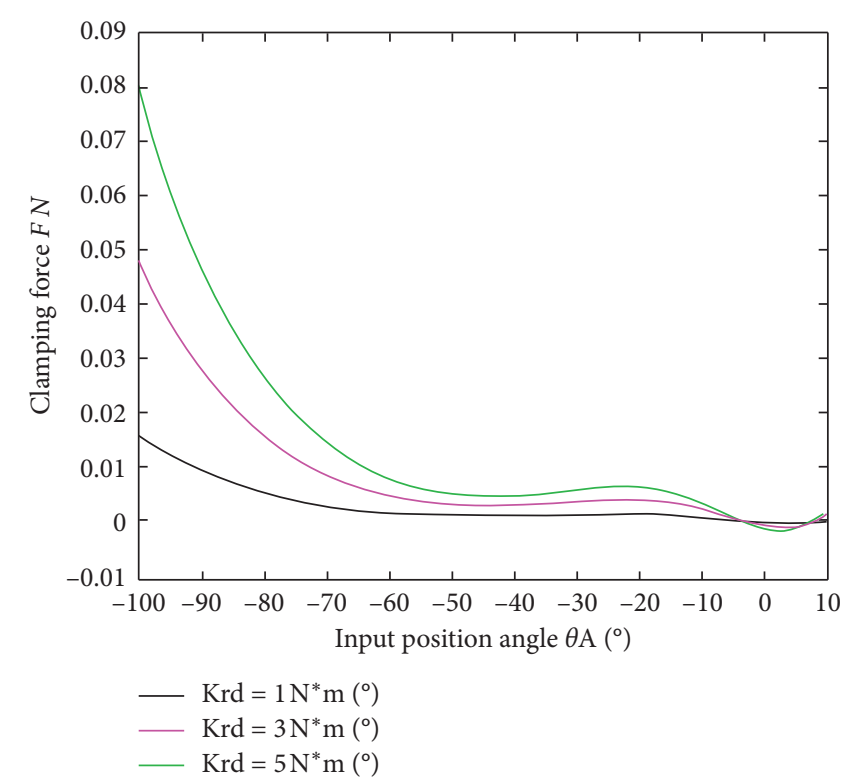

(b)

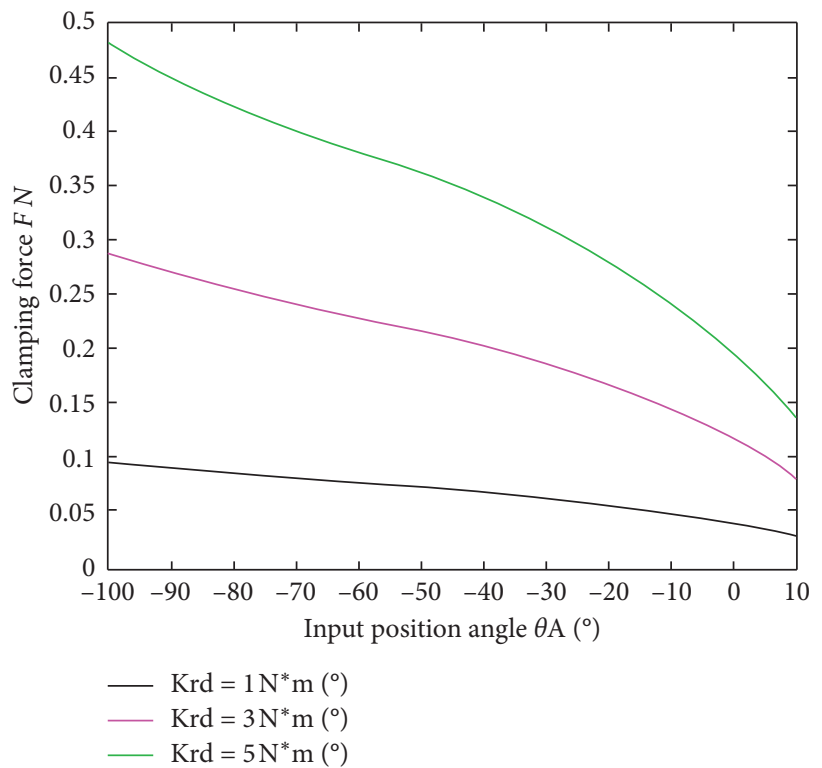

(d)

Figure 7: Grasping force $F$ changes with position angle $\theta_{A}$.

Using this optimization method, the appropriate spring stiffness can be found.

It can be seen from the previous section that, $K_{R A}, K_{R C}$, and $K_{R D}$ all produce monotonic increasing curve, and $T_{d}$ generates bistable curve. Based on image analysis, if a constant force is required, it can be assumed that $K_{R A}=$ $K_{R C}=0$ and $T_{d}=1 \mathrm{~N} \cdot \mathrm{m}$, then $K_{R D}=5.2741 \mathrm{~N} \cdot \mathrm{cm} /{ }^{\circ}$. At this time, the grasping force is

$$
F=\frac{1-5.2741\left(\theta_{D}-\theta_{D 0}\right)\left(\mathrm{d} \theta_{D} / \mathrm{d} \theta_{A}\right)}{r_{5} \sin \left(100^{\circ}-\theta_{D}\right)\left(\mathrm{d} \theta_{D} / \mathrm{d} \theta_{A}\right)}
$$

This is shown in Figure 8.
It can be seen from the image that $\theta_{A}=-50^{\circ}$ is in the constant force range. Because the actual gripping force is $167 \mathrm{~N}$, the gripping force is set to $180 \mathrm{~N}$ for safety reasons. According to formula (27), the required torque $T_{d}$ can be obtained as follows:

$$
\begin{aligned}
T_{d} & =\frac{180 \cdot r_{5} \sin \left(100^{\circ}-\theta_{D}\right)\left(\mathrm{d} \theta_{D} / \mathrm{d} \theta_{A}\right)}{1+5.2741\left(\theta_{D}-1.4415\right)\left(\mathrm{d} \theta_{D} / \mathrm{d} \theta_{A}\right)} \\
& =720.5607 \mathrm{~N} \cdot \mathrm{m} \approx 720 \mathrm{~N} \cdot \mathrm{m} .
\end{aligned}
$$

At this time, we can get $K_{R D}=37.97352 \mathrm{~N} \cdot \mathrm{m} /{ }^{\circ}$. At this time, the grasping force is 


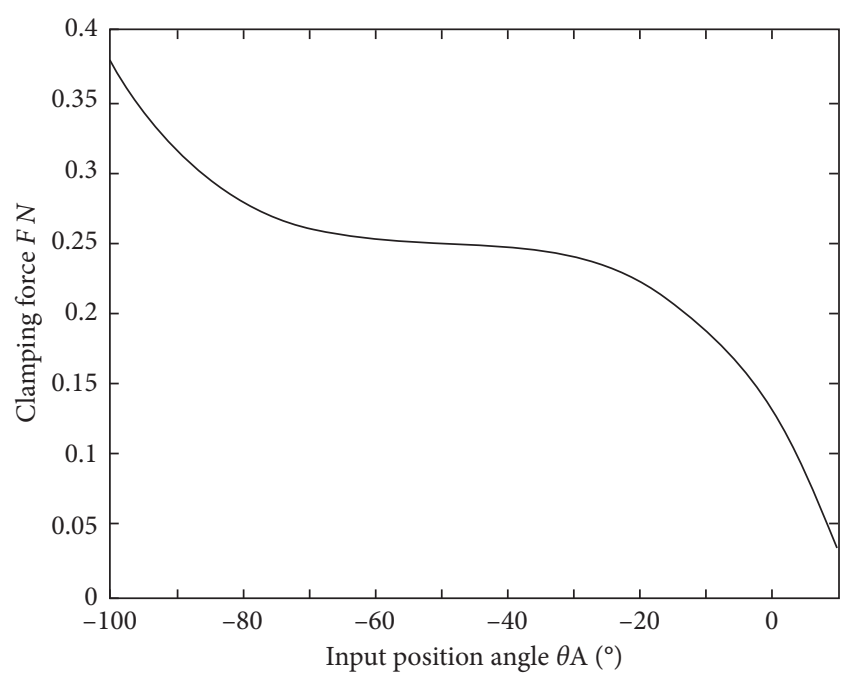

Figure 8: Grasping force $F$ changes with position angle $\theta_{A}$.

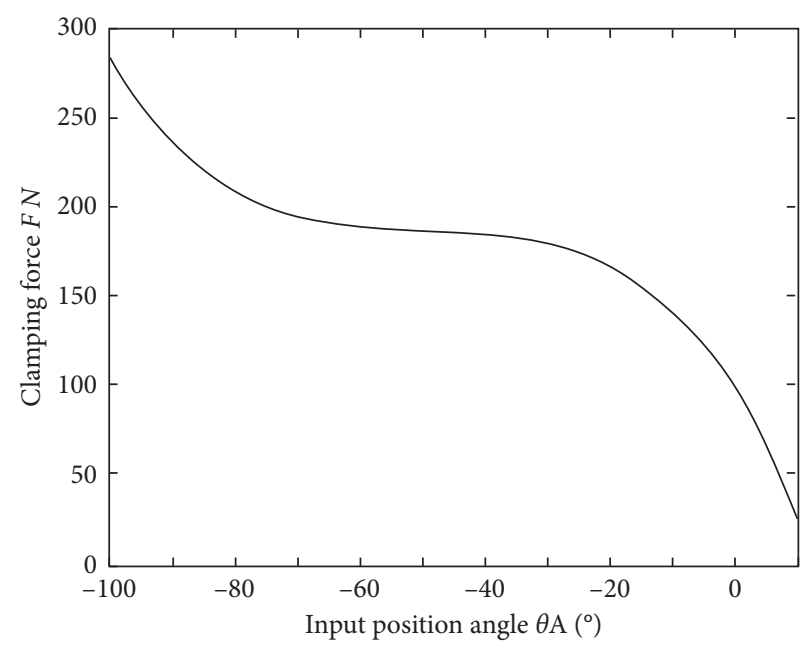

Figure 9: Grasping force $F$ changes with position angle $\theta_{A}$.

$$
F=\frac{T_{d}-K_{R D}\left(\theta_{D}-\theta_{D 0}\right)\left(\mathrm{d} \theta_{D} / \mathrm{d} \theta_{A}\right)}{r_{5} \sin \left(100^{\circ}-\theta_{D}\right)\left(\mathrm{d} \theta_{D} / \mathrm{d} \theta_{A}\right)}
$$

This is shown in Figure 9.

According to the equation, the search constant force interval is [-64.2317, -34.99948$]$. In this interval, grasping force $F$ can be regarded as constant force. Through the calculation and analysis of the mechanism, the range of constant force interval can fully meet the requirement of grasping tyres with inner diameter ranging from $404.9 \mathrm{~mm}$ to $606.4 \mathrm{~mm}$. As long as the position of the mechanism is properly adjusted, the tyres with different inner diameters can be grasped with constant force.

The grabbing force of the improved mechanism changes with the angle is as shown in Figure 10.

As can be seen from Figure 10, the grip force of the improved mechanism is very unstable. With the change of the inner diameter of the tyre, it is easy to appear that the grip force is too strong to cause the deformation of the tyre. As can be seen from the previous section, the improved grip

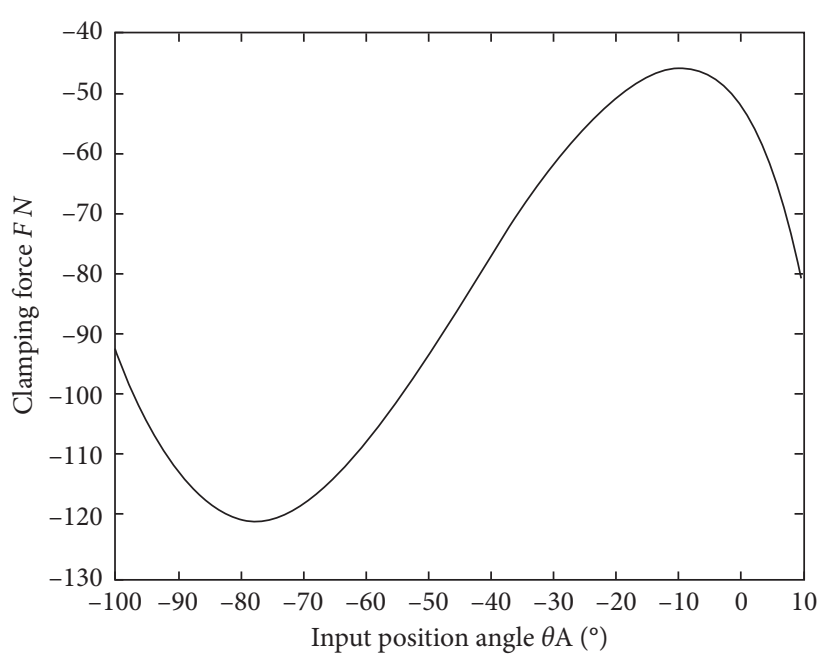

FIgURE 10: Grasping force $F$ changes with position angle $\theta_{A}$.

force has little change and can be regarded as constant grip force.

\section{Conclusion}

In this paper, a manipulator of tyre constant force grasping mechanism is designed by using a spring stiffness design, and the nonlinear stiffness characteristics of crank-rocker mechanism are modeled and analyzed. The following conclusions can be drawn: (1) By designing the spring stiffness of the connecting rod mechanism nodes, the connecting rod mechanism can be transformed from a rigid-body mechanism to a pseudorigid-body mechanism, which greatly reduces the clearance and friction of the mechanism and increases the accuracy and stability of the mechanism. (2) By modeling and analyzing the nonlinear stiffness characteristics of the mechanism, the expected nonlinear stiffness can be obtained, and the clamping mechanism of the manipulator can be improved into a constant force clamping mechanism. (3) Compared with the general constant force mechanism, the design and calculation of the four-bar constant force mechanism are more complicated. Because of the problems of multiple dead points, the virtual work principle should be used to analyze the mechanism rather than the Lagrange equation. (4) Because of the more complex structure of the four-bar mechanism, it is difficult to achieve constant force in the process of motion. It can only design a constant force interval of one or several segments, and the range of the constant force interval is also affected by the length of each link. Therefore, the scope of adaptation will be affected.

\section{Data Availability}

The data used to support the findings of this study are available from the corresponding author upon request.

\section{Conflicts of Interest}

The authors declare that they have no conflicts of interest. 


\section{Acknowledgments}

This work was supported by the Natural Science Foundation of China (Grant no. 51705268).

\section{References}

[1] P. Wang and Q. Xu, "Design and modeling of constant-force mechanisms: a survey," Mechanism and Machine Theory, vol. 119, pp. 1-21, 2018.

[2] G. Chen, H. Chang, and L. Geng, "Design of constant-force compliant Sarrus mechanism considering stiffness nonlinearity of compliant joints," in Advances in Reconfigurable Mechanisms and Robots II, vol. 36, pp. 107-116, Springer, Cham, Switzerland, 2016.

[3] C.-W. Hou and C.-C. Lan, "Functional joint mechanisms with constant-torque outputs," Mechanism and Machine Theory, vol. 62, pp. 166-181, 2013.

[4] L. Patrice and J. L. Herder, "An adjustable constant force mechanism using pin joints and springs," New Trends in Mechanism and Machine Science, vol. 43, pp. 453-461, Springer, Cham, Switzerland, 2017.

[5] P. Wang and Q. Xu, "Design of a compact compliant constant-force XY precision positioning stage," in Proceedings of the 2016 12th IEEE/ASME International Conference on Mechatronic and Embedded Systems and Applications (MESA), Auckland, New Zealand, 2016.

[6] K. A. Tolman, E. G. Merriam, and L. L. Howell, "Compliant constant-force linear-motion mechanism," Mechanism and Machine Theory, vol. 106, pp. 68-79, 2016.

[7] Y. Liu, Y. Zhang, and Q. Xu, "Design and control of a novel compliant constant-force gripper based on buckled fixedguided beams," IEEE/ASME Transactions on Mechatronics, vol. 22, no. 1, pp. 476-486, 2016.

[8] A. Midha, L. L. Howell, and T. W. Norton, "Limit positions of compliant mechanisms using the pseudo-rigid-body model concept," Mechanism and Machine Theory, vol. 35, no. 1, pp. 99-115, 2000.

[9] C. Boyle, L. L. Howell, S. P. Magleby, and M. S. Evans, "Dynamic modeling of compliant constant-force compression mechanisms," Mechanism and Machine Theory, vol. 38, no. 12, pp. 1469-1487, 2003.

[10] Y. Zheng, Q. Li, B. Yan, Y. Luo, and X. Zhang, "A Stewart isolator with high-static-low-dynamic stiffness struts based on negative stiffness magnetic springs," Journal of Sound and Vibration, vol. 422, pp. 390-408, 2018.

[11] Z.-W. Yang and C.-C. Lan, "An adjustable gravity-balancing mechanism using planar extension and compression springs," Mechanism and Machine Theory, vol. 92, pp. 314-329, 2015.

[12] B. Andò, S. Baglio, A. R. Bulsara, and V. Marletta, "A bistable buckled beam based approach for vibrational energy harvesting," Sensors and Actuators A: Physical, vol. 211, pp. 153-161, 2014.

[13] L. de Haro Silva, P. J. Paupitz Gonçalves, D. Wagg, and W. David, "On the dynamic behavior of the Zener model with nonlinear stiffness for harmonic vibration isolation," $\mathrm{Me}$ chanical Systems and Signal Processing, vol. 112, pp. 343-358, 2018.

[14] Z. Wu, C. Levi, and S. F. Estefen, "Wave energy harvesting using nonlinear stiffness system," Applied Ocean Research, vol. 74, pp. 102-116, 2018.

[15] I. Kovacic, M. J. Brennan, and T. P. Waters, "A study of a nonlinear vibration isolator with a quasi-zero stiffness characteristic," Journal of Sound and Vibration, vol. 315, no. 3, pp. 700-711, 2008.

[16] B. Tang, M. J. Brennan, G. Gatti, and N. S. Ferguson, "Experimental characterization of a nonlinear vibration absorber using free vibration," Journal of Sound and Vibration, vol. 367, pp. 159-169, 2016.

[17] S. P. Pellegrini, N. Sergio, M. Schenk, and J. L. Herder, "Bistable vibration energy harvesters: a review," Journal of Intelligent Material Systems and Structures, vol. 24, no. 11, pp. 1303-1312, 2013.

[18] X. Tolou and X. Jing, "Analysis and design of a nonlinear stiffness and damping system with a scissor-like structure," Mechanical Systems and Signal Processing, vol. 66-67, pp. 723-742, 2016.

[19] S. Wang et al., "Neural-network-based adaptive funnel control for servo mechanisms with unknown dead-zone," IEEE transactions on cybernetics, vol. 50, no. 4, pp. 1383-1394, 2018.

[20] S. Wang, J. Na, X. Ren, H. Yu, and J. Yu, "Unknown input observer-based robust adaptive funnel motion control for nonlinear servomechanisms," International Journal of Robust and Nonlinear Control, vol. 28, no. 18, pp. 6163-6179, 2018.

[21] S. Wang, H. Yu, and J. Yu, "Robust adaptive tracking control for servo mechanisms with continuous friction compensation," Control Engineering Practice, vol. 87, pp. 76-82, 2019.

[22] P. Alabuzhev, A. Gritchin, and L. Kim, Vibration Protecting and Measuring Systems with Quasi-Zero Stiffness, Hemisphere Publishing Corp, New York, NY, USA, 1989.

[23] W. B. Lewis, "Development and Design of Constant-Force Mechanisms," Doctoral Dissertation, Brigham Young University, Provo, UT, USA, 2002.

[24] B. Li and G. Hao, "Nonlinear behaviour design using the kinematic singularity of a general type of double-slider fourbar linkage," Mechanism and Machine Theory, vol. 129, pp. 106-130, 2018.

[25] X.-B. Chen, N. Iwatsuki, and I. Hayashi, "Kinematic analysis at singular points of linkages based on L'hospital's theorem," Journal of Tongji University, vol. 30, no. 8, pp. 973-978, 2002.

[26] B. Li and G. Hao, "On generating expected kinetostatic nonlinear stiffness characteristics by the kinematic limbsingularity of a crank-slider linkage with springs," Chinese Journal of Mechanical Engineering, vol. 32, no. 1, pp. 32-54, 2019. 